\title{
Tamanho de amostra para a estimação da média do comprimento, diâmetro e massa de sementes de feijão de porco e mucuna cinza
}

\author{
Sample size for estimating the average length, diameter and weight of seeds of jack bean \\ and velvet bean
}

\author{
Alberto Cargnelutti Filho ${ }^{\mathrm{I}}$ Marcos Toebe ${ }^{\mathrm{II}}$ Cláudia Burin ${ }^{\mathrm{III}}$ André Luis Fick ${ }^{\mathrm{III}}$ \\ Bruna Mendonça Alves ${ }^{\mathrm{II}}$ Giovani Facco ${ }^{\mathrm{IV}}$
}

\begin{abstract}
O objetivo deste trabalho foi determinar o tamanho de amostra necessário para a estimação da média do comprimento, dos diâmetros maior e menor e da massa de sementes de feijão de porco (Canavalia ensiformis) e de mucuna cinza (Stizolobium cinereum). Em 300 sementes de feijão de porco e em 300 sementes de mucuna cinza, foram mensurados os seguintes caracteres: comprimento, diâmetros maior e menor e massa. Foram calculadas medidas de tendência central e de variabilidade. Após, foram testadas as hipóteses de igualdade entre as médias e de homogeneidade entre as variâncias. Foi determinado o tamanho de amostra por meio de reamostragem, com reposição de 10.000 amostras. Para a estimação da média do comprimento, dos diâmetros maior e menor e da massa, com intervalo de confiança de 95\% igual a 10\% da estimativa da média, 117 e 66 sementes são suficientes, respectivamente, para feijão de porco e mucuna cinza.
\end{abstract}

Palavras-chave: Canavalia ensiformis, Stizolobium cinereum, planejamento experimental, reamostragem.

\section{ABSTRACT}

The objective of this research was to determine the sample size (number of seeds) to estimate the average of length, major and minor diameters and weight of seeds of jack bean (Canavalia ensiformis) and velvet bean (Stizolobium cinereum).
In 300 seeds of jack bean and 300 seeds of velvet bean it was measured following characters: length, major and minor diameters and weight. It was calculated measures of central tendency and variability. After the hypothesis of equality between the means and homogeneity of variances, were tested. It was determined the sample size using resampling with replacement of 10,000 samples. For estimating the average of length, major and minor diameters and weight, with amplitude of confidence interval of 95\%, equal 10\% of average estimate, 117 and 66 seeds are sufficient, respectively, for jack bean and velvet bean.

Key words: Canavalia ensiformis, Stizolobium cinereum, experimental design, resampling.

As espécies leguminosas possuem capacidade de fixação biológica de nitrogênio e ciclagem de nutrientes, sendo utilizadas no sistema de rotação de culturas. Segundo TEODORO et al. (2011), as culturas de feijão de porco e de mucuna cinza estão entre as espécies leguminosas mais promissoras para a adubação verde, devido à elevada produção de matéria seca. Além disso, TEODORO et al. (2011) relatam que, aos 40 dias, o feijão de porco e a mucuna cinza já cobrem o solo, com 67 e 63\%, respectivamente, favorecendo a sua inserção nos sistemas de cultivo. Ainda, após 16 anos de utilização

IDepartamento de Fitotecnia, Centro de Ciências Rurais (CCR), Universidade Federal de Santa Maria (UFSM), 97105-900, Santa Maria, RS, Brasil. E-mail: alberto.cargnelutti.filho@gmail.com. Autor para correspondência.

IIPrograma de Pós-graduação em Agronomia, UFSM, Santa Maria, RS, Brasil.

IIICurso de Engenharia Florestal, UFSM, Santa Maria, RS, Brasil.

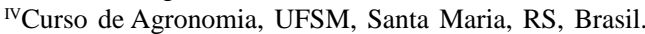


de sete sistemas de culturas, LANZANOVA et al. (2010) verificaram que o uso das culturas de feijão de porco e de mucuna cinza foram eficientes em manter as condições físicas do solo (densidade, porosidade, resistência à penetração e taxa de infiltração de água) adequadas ao desenvolvimento vegetal.

Em pesquisas sobre as sementes dessas culturas, é importante dimensionar o tamanho de amostra (número de sementes) adequado para avaliar caracteres como: comprimento, diâmetros maior e menor e massa, com precisão desejada. Na situação de maior variabilidade dos dados desses caracteres, maior confiabilidade desejada na estimativa e menor erro de estimação permitido a priori pelo pesquisador, será necessário maior tamanho de amostra (SPIEGEL et al., 2004).

Para o estudo do dimensionamento do tamanho de amostra, o uso de intervalos de confiança, obtidos por reamostragem, é adequado (FERREIRA, 2009). Essa técnica independe da distribuição de probabilidade dos dados e já foi utilizada na determinação do tamanho de amostra para a avaliação de famílias de irmãos completos em cana-de-açúcar (LEITE et al., 2009) e para a estimação do coeficiente de correlação linear de Pearson entre caracteres de milho (CARGNELUTTI FILHO et al., 2010). Contudo, estudos que determinem o tamanho de amostra necessário para avaliar caracteres de sementes de feijão de porco e de mucuna cinza não foram encontrados na literatura. Assim, o objetivo deste trabalho foi determinar o tamanho de amostra necessário para a estimação da média do comprimento, dos diâmetros maior e menor e da massa de sementes de feijão de porco e de mucuna cinza.

A partir de oito kg de sementes de feijão de porco, provenientes de um lote composto por 344 embalagens de $20 \mathrm{~kg}$ (6.880kg), com 99,0\% de sementes puras e com $70 \%$ de germinação, foram tomadas, aleatoriamente, 300 sementes. Também, a partir de oito $\mathrm{kg}$ de sementes de mucuna cinza, provenientes de um lote composto por 160 embalagens de $50 \mathrm{~kg}$ (8.000kg), com $99,8 \%$ de sementes puras e com $95 \%$ de germinação, foram tomadas, aleatoriamente, 300 sementes. Em cada uma dessas 300 sementes, de cada cultura, foram mensurados os seguintes caracteres: comprimento, diâmetros maior e menor, com o auxílio de um paquímetro e massa com uma balança digital, com precisão de $0,001 \mathrm{~g}$. As mensurações foram realizadas no Departamento de Fitotecnia da Universidade Federal de Santa Maria, em 2010.
Para cada caractere de cada cultura, foram calculadas medidas de tendência central e de variabilidade. Foram aplicados os testes t (bilateral) e F (bilateral), a 5\% de probabilidade de erro, para testar, respectivamente, as hipóteses de igualdade entre as médias e de homogeneidade entre as variâncias, de cada um dos quatro caracteres mensurados nas sementes de feijão de porco e de mucuna cinza.

A seguir, para as simulações, foram planejados 299 tamanhos de amostra para cada caractere de cada cultura, sendo o tamanho de amostra inicial de duas sementes, e os demais obtidos com incrementos de uma até atingir 300 sementes. Portanto, para as simulações, foram planejados os seguintes tamanhos de amostra: 2, 3, 4, ..., 300 sementes para cada caractere de cada cultura.

Após, para cada tamanho de amostra planejado de cada caractere de cada cultura, foram realizadas 10.000 simulações, por meio de reamostragem, com reposição. Para cada amostra simulada, foi estimada a média. Assim, para cada tamanho de amostra de cada caractere de cada cultura, foram obtidas 10.000 estimativas da média (FERREIRA, 2009) e determinados os valores mínimo, percentil 2,5\%, média, percentil 97,5\% e máximo. Depois, calculou-se a amplitude do intervalo de confiança de $95 \%$ pela diferença entre o percentil $97,5 \%$ e o percentil 2,5\%. A seguir, determinouse o tamanho de amostra (número de sementes) para a estimação da média de cada caractere de cada cultura. Para essa determinação, partiu-se do tamanho inicial (duas sementes) e considerou-se como tamanho de amostra o número de sementes a partir do qual a amplitude do intervalo de confiança de $95 \%$ foi menor ou igual a $10 \%$ da estimativa da média.

Os valores mínimo, percentil 2,5\%, média, percentil 97,5\% e máximo dos tamanhos de amostra (2, $7,12, \ldots, 297$ sementes) da massa de sementes de cada cultura foram plotados em gráficos. A opção de representar graficamente apenas a massa de sementes foi em função de a massa necessitar maior tamanho de amostra para a estimação da média. Optou-se pela representação gráfica, com intervalo de cinco sementes, pela melhor representação visual. As análises estatísticas foram realizadas com o auxílio do programa R (R DEVELOPMENT CORE TEAM, 2011) e do aplicativo Microsoft Office Excel.

As estatísticas descritivas revelaram existência de variabilidade entre as sementes de feijão de porco (Canavalia ensiformis) e de mucuna cinza (Stizolobium cinereum) (Tabela 1). As sementes de 
Tabela 1 - Mínimo, máximo, média, mediana, amplitude, variância, desvio padrão, erro padrão e coeficiente de variação (CV) do comprimento (COMP), dos diâmetros maior (DMA) e menor (DME) e da massa (MASSA) de 300 sementes de feijão de porco (Canavalia ensiformis) e de 300 sementes de mucuna cinza (Stizolobium cinereum).

\begin{tabular}{|c|c|c|c|c|c|c|c|c|}
\hline Estatística & $\begin{array}{c}\text { COMP } \\
(\mathrm{cm})\end{array}$ & $\begin{array}{l}\text { DMA } \\
(\mathrm{cm})\end{array}$ & $\begin{array}{l}\text { DME } \\
(\mathrm{cm})\end{array}$ & $\begin{array}{c}\text { MASSA } \\
\text { (g) }\end{array}$ & $\begin{array}{c}\text { COMP } \\
(\mathrm{cm})\end{array}$ & $\begin{array}{l}\text { DMA } \\
(\mathrm{cm})\end{array}$ & $\begin{array}{l}\text { DME } \\
(\mathrm{cm})\end{array}$ & $\begin{array}{c}\text { MASSA } \\
\text { (g) }\end{array}$ \\
\hline Mínimo & 1,300 & 0,900 & 0,550 & 0,500 & 1,150 & 0,900 & 0,550 & 0,460 \\
\hline Máximo & 2,200 & 1,450 & 1,050 & 1,958 & 1,900 & 1,450 & 1,000 & 1,642 \\
\hline Média & $1,769 \mathrm{a}$ & $1,180 b$ & $0,825 b$ & $1,150 \mathrm{a}$ & $1,580 \mathrm{~b}$ & $1,216 a$ & $0,841 \mathrm{a}$ & $1,125 a$ \\
\hline Mediana & 1,800 & 1,200 & 0,800 & 1,128 & 1,600 & 1,200 & 0,850 & 1,153 \\
\hline Amplitude & 0,900 & 0,550 & 0,500 & 1,458 & 0,750 & 0,550 & 0,450 & 1,182 \\
\hline Variância & $0,031 \mathrm{a}$ & $0,013 a$ & 0,010a & $0,101 \mathrm{a}$ & $0,019 b$ & $0,008 \mathrm{~b}$ & $0,004 b$ & $0,053 b$ \\
\hline Desvio padrão & 0,175 & 0,116 & 0,098 & 0,317 & 0,139 & 0,091 & 0,060 & 0,231 \\
\hline Erro padrão & 0,010 & 0,007 & 0,006 & 0,018 & 0,008 & 0,005 & 0,003 & 0,013 \\
\hline CV (\%) & 9,921 & 9,822 & 11,889 & 27,593 & 8,803 & 7,479 & 7,075 & 20,498 \\
\hline
\end{tabular}

${ }^{(1)}$ As médias de cada caractere mensurado em sementes de feijão de porco e de mucuna cinza, não seguidas pela mesma letra, diferem pelo teste t bilateral, a $5 \%$ de probabilidade de erro.

${ }^{(2)}$ As variâncias de cada caractere mensurado em sementes de feijão de porco e de mucuna cinza, não seguidas pela mesma letra, são heterogêneas pelo teste $\mathrm{F}$ bilateral, a 5\% de probabilidade de erro.

feijão de porco apresentaram maior comprimento e menores diâmetros em relação às sementes de mucuna cinza. Isso, provavelmente, explica a não diferença de massa entre as sementes dessas culturas. As variâncias entre as culturas (feijão de porco e mucuna cinza) foram heterogêneas para os caracteres comprimento, diâmetros maior e menor e massa, o que indica tamanho de amostra diferenciado entre as culturas.

A massa de sementes apresentou maior coeficiente de variação ( $C V=27,593 \%$ e $C V=20,498 \%$ ), nas culturas de feijão de porco e de mucuna cinza, respectivamente, em relação aos caracteres comprimento, diâmetros maior e menor $(7,075 \% \leq \mathrm{CV} \leq 11,889 \%)$. Esses resultados sugerem maiores tamanhos de amostra para a estimação da média da massa de sementes em relação aos demais caracteres, nas duas culturas.

Os tamanhos de amostra (número de sementes) para a estimação da média da massa de sementes de feijão de porco e de mucuna cinza, para uma amplitude do intervalo de confiança de 95\% igual a $10 \%$ da estimativa da média, foram, respectivamente, 117 e 66 sementes (Figura 1). Para a estimação da média do comprimento e dos diâmetros maior e menor de feijão de porco, com intervalo de confiança de 95\% igual a $10 \%$ da estimativa da média, os tamanhos de amostra foram, respectivamente, 16, 16 e 23 sementes. Já para estimação da média do comprimento e dos diâmetros maior e menor de mucuna cinza, com intervalo de confiança de $95 \%$ igual a $10 \%$ da estimativa da média, os tamanhos de amostra foram, respectivamente, 13, 10 e 9 sementes. Portanto, esses resultados reforçam inferências supracitadas sobre a necessidade de maior tamanho de amostra para avaliar a massa de sementes em relação ao comprimento e aos diâmetros maior e menor, nessas duas culturas. Embora, nas pesquisas de LEITE et al. (2009) e CARGNELUTTI FILHO et al. (2010), a reamostragem tenha sido utilizada, não foram encontrados, em sementes, trabalhos que pudessem ser utilizados para a comparação desses resultados, com essa metodologia.

Em sementes de feijão de porco e de mucuna cinza, 117 e 66 sementes, respectivamente, são suficientes para a estimação do comprimento, dos diâmetros maior e menor e da massa, com intervalo de confiança de $95 \%$ igual a $10 \%$ da estimativa da média.

\section{AGRADECIMENTOS}

Ao Conselho Nacional de Desenvolvimento Científico e Tecnológico (CNPq) e à Coordenação de Aperfeiçoamento de Pessoal de Nível Superior (CAPES), pelas bolsas concedidas. À Fundação de Amparo à Pesquisa do Estado do Rio Grande do Sul, pelo auxílio financeiro. 


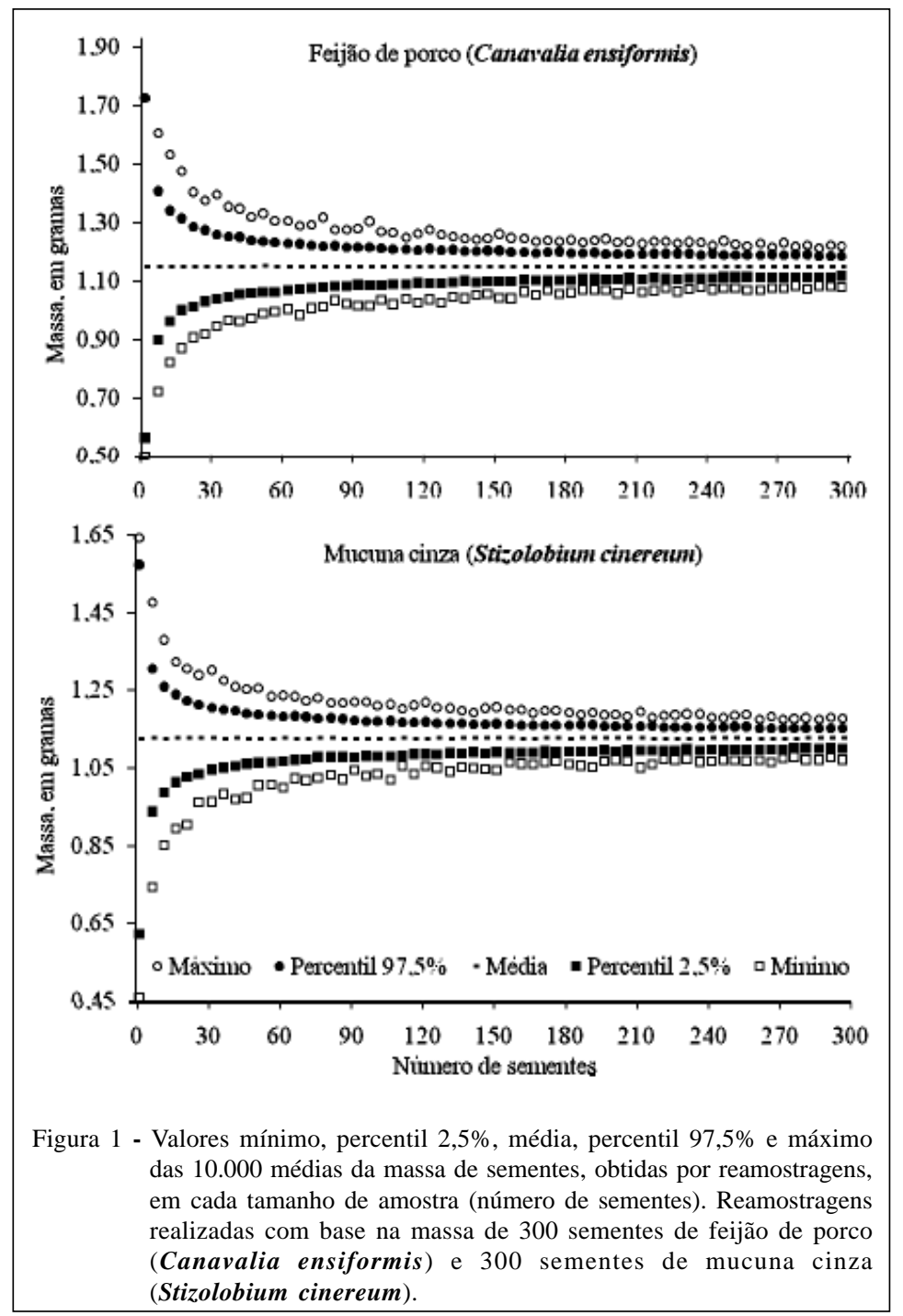

\section{REFERÊNCIAS}

CARGNELUTTI FILHO, A. et al. Tamanho de amostra para estimação do coeficiente de correlação linear de Pearson entre caracteres de milho. Pesquisa Agropecuária Brasileira, v.45, p.1363-1371, 2010. Disponível em: <http://www.scielo.br/ pdf/pab/v45n12/v45n12a05.pdf>. Acesso em: 22 dez. 2011. doi: 10.1590/S0100-204X2010001200005.

FERREIRA, D.F. Estatística básica. 2.ed. Lavras: UFLA, 2009. 664p.

LANZANOVA, M.E. et al. Atributos físicos de um argissolo em sistemas de culturas de longa duração sob semeadura direta. Revista Brasileira de Ciência do Solo, v.34, p.1333-1342, 2010. Disponível em: <http://www.scielo.br/pdf/rbcs/v34n4/ 30.pdf $>$. Acesso em: $22 \mathrm{dez}$. 2011. doi: 10.1590/S010006832010000400030 .
LEITE, M.S.O. et al. Sample size for full-sib family evaluation in sugarcane. Pesquisa Agropecuária Brasileira, v.44, p. 1562-1574, 2009. Disponível em: <http://www.scielo.br/pdf/ $\mathrm{pab} / \mathrm{v} 44 \mathrm{n} 12 / \mathrm{v} 44 \mathrm{n} 12 \mathrm{a} 02 . \mathrm{pdf}>$. Acesso em: $22 \mathrm{dez}$. 2011. doi: 10.1590/S0100-204X2009001200002.

R DEVELOPMENT CORE TEAM. R: a language and environment for statistical computing. Vienna: R Foundation for Statistical Computing, 2011. Disponível em: <http:// biostat.mc.vanderbilt.edu/wiki/pub/Main/SweaveLatex/ RCitation.pdf>. Acesso em: 13 mar. 2011.

SPIEGEL, R.A. et al. Probabilidade e estatística. 2.ed. Porto Alegre: Bookman, 2004. 398p.

TEODORO, R.B. et al. Aspectos agronômicos de leguminosas para adubação verde no Cerrado do Alto Vale do Jequitinhonha. Revista Brasileira de Ciência do Solo, v.35, p.635-640, 2011. Disponível em: <http://www.scielo.br/pdf/rbcs/v35n2/ v35n2a32.pdf>. Acesso em: 22 dez. 2011. doi: 10.1590/S010006832011000200032 . 